\title{
O conhecimento da equipe de enfermagem no uso de protocolos para atendimento de paciente politraumatizado
}

RESUMO | Objetivo: Analisar as representações sociais da equipe de enfermagem sobre o conhecimento técnico científico dos protocolos para atendimento de paciente politraumatizados. Método: pesquisa de campo e qualitativa com dados coletados por entrevistas semiestruturadas áudio-gravadas no mês de dezembro de 2019. Resultados: A análise dos dados obtidos permitiram construir seis discursos a partir das expressões-chave, com suas respectivas ideias centrais. Considerações Finais: A equipe de enfermagem apesar de realizar algumas das etapas do protocolo preconizado pela instituição, de forma assistemática, têm dificuldades em reconhecer e o significado de protocolos.

Palavras-chaves: Cuidados de Enfermagem; Ferimentos e lesões; Equipe de enfermagem; Protocolos de enfermagem.

\begin{abstract}
Objective: To analyze the social representations of the nursing team about the technical scientific knowledge of the protocols for the care of polytrauma patients. Method: field and qualitative research with data collected by audio-recorded semi-structured interviews in December 2019. Results: The analysis of the data obtained allowed the construction of six speeches based on the key expressions, with their respective central ideas. Final Considerations: Although the nursing team performs some of the steps of the protocol recommended by the institution, in an unsystematic way, they have difficulties in recognizing and the meaning of protocols.
\end{abstract}

Keywords: Nursing Care; Wounds and Injuries; Nursing, Team; Nursing Assessment,

RESUMEN | Objetivo: Analizar las representaciones sociales del equipo de enfermería sobre el conocimiento científico técnico de los protocolos para la atención de pacientes politraumatizados. Método: investigación de campo y cualitativa con datos recolectados por entrevistas semiestructuradas grabadas en audio en diciembre de 2019. Resultados: El análisis de los datos obtenidos permitió la construcción de seis discursos a partir de las expresiones clave, con sus respectivas ideas centrales. Consideraciones finales: Si bien el equipo de enfermería realiza algunos de los pasos del protocolo recomendados por la institución, de manera no sistemática, tienen dificultades para reconocer y el significado del protocolos.

Palabras claves: Atención de Enfermería, Heridas y Traumatismos; Grupo de Enfermería, Evaluación en Enfermería.

\section{Juliana Kaori Ikeda Loureiro}

Enfermeira. Residente em Saúde da Família, Escola de Saúde Pública de Florianópolis. Florianópolis (SC), Brasil.

ORCID: 0000-0001-5091-3615

\section{Joyce Fernanda Soares Albino Ghezzi}

Docente do curso de Enfermagem da Faculdade de Ensino Superior e Formação Integral - FAEF. Marília (SP), Brasil.

ORCID: 000-0002-5808-613X

\section{Shirlene Pavelqueires}

Professora Doutora da Disciplina de Enfermagem Clínica da Faculdade de Medicina de Marília- FAMEMA e Professora Doutora do Curso de Medicina da Fundação Educacional Municipal de Assis- FEMA. Vera Cruz (SP), Brasil.

ORCID: 0000-0002-8740-351X

\section{Elza de Fátima Ribeiro Higa}

Docente da Faculdade de Medicina de Marília. ORCID: 0000-0001-5772-9597

Recebido em: 04/05/2021

Aprovado em: 25/05/2021

\section{INTRODUÇÃO}

$\Lambda$ nualmente nota-se o aumento no percentil de mortalidade advindos do trauma, visto que este é o principal fator de óbito entre jovens abaixo de 44 anos de idade e somados a violência atinge o valor de 5,8 milhões de vítimas no mundo ${ }^{(1)}$. Esse público encontra-se, muitas vezes, com instabilidade fisiológica e consequentemente mais vulnerável a contrariedade de eventos, portanto, o mau manejo de pacientes politraumatizados por falta de conhecimento e a falha na execução dos protocolos, acarreta piora ou lesões que poderiam ser evitadas. Nesse sentido, faz-se primordial uma equipe competente para reconhecer lesões e evitar possíveis danos nas vítimas de politraumatismo ${ }^{(2)}$.

Estudo realizado nos Estados Unidos mostrou que a prática segura tem sido tema de significativa relevância enfatizando o número exorbitante de mortes preveníveis por ano devido a erros na atenção à saúde ${ }^{(3)}$.

Quando uma equipe recebe orientações e capacitações, apresentam resultados mais satisfatórios, o que releva a importância da realização constante da educação permanente e continuada. E, levando em consideração que a equipe de enfermagem representa mais da metade dos profissionais de saúde no Brasil, justifica-se a precisão de estudos científicos que reflitam em melhorias nos cuidados de enfermagem dispensados aos pacientes politraumatizados ${ }^{(4-5)}$.

Em revisão de literatura, denotou-se indispensabilidade do conhecimento básico dos enfermeiros para compreender as prioridades dos pacientes vítimas de trauma assim como sua atualização através de estudos clínicos que favorecem a Prática Baseada em Evidência (PBE). Para tal, recomenda-se práticas fundamentadas 
em protocolos como por exemplo o Advanced Trauma Life Support (ATLS) e Prehospital Trauma Life Support (PHTLS) ${ }^{(6)}$.

Os protocolos, além de serem ferramentas teóricas que potencializam a qualidade da assistência, minimizam a suscetibilidade de erros e contribuírem para a padronização dos serviços, favorecendo assim, resultados positivos e práticas mais seguras ${ }^{(7)}$.

Tendo em vista a importância da equipe de enfermagem atuar com práticas pautadas em protocolos, com vistas a reduzir mortalidades causadas por atuações empíricas, esta pesquisa se estruturou a partir do seguinte questionamento: quais os conhecimentos técnico-científicos sobre protocolos de atendimento aos pacientes politraumatizados de uma equipe de enfermagem atuante em um serviço de urgência e emergência de um hospital de médio porte do interior paulista? Teve, portanto, como objetivo analisar as representações sociais da equipe de enfermagem sobre o conhecimento técnico científico dos protocolos para atendimento de paciente politraumatizados.

\section{MÉTODO}

Pesquisa de campo e qualitativa pautada nos pressupostos da Teoria das Representações Sociais (TRS), com dados coletados por meio de entrevistas semiestruturadas e registrados por áudio-gravação.

Para o desenvolvimento da pesquisa, seguiu-se as exigências estabelecidas pelo COREQ Guideline, o qual direcionada os critérios consolidados de uma pesquisa qualitativa ${ }^{(8)}$.

O cenário de pesquisa foi um serviço de emergência de um hospital de médio porte, localizado no interior paulista. A estruturação deste serviço é composta por sala de emergência com 10 leitos, para os pacientes em geral, e um leito, para paciente com necessidade de isolamento. Ademais, o serviço contempla uma área externa para desembarque de ambulância. Este hospital atua com assistência de média e alta complexidade e diversas especialidades, sendo referência para 62 municípios $^{(9)}$.

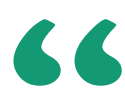

Para discussão dos dados utilizou-se por referencial teórico a Teoria das Representações Sociais (TRS). As representações sociais são fenômenos que se relacionam com a forma singular de compreender e de se comunicar, o qual concebe tanto a realidade quanto o senso comum.

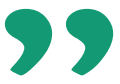

Foram convidados a participar da pesquisa todos os profissionais de enfermagem de nível médio ou superior, atuante na sala de emergência. A população geral corresponde ao total de
11 enfermeiros e 56 técnicos/auxiliares de enfermagem. A escolha dessa categoria profissional se apoiou no fato desta representar a maior classe profissional no local de pesquisa.

Os dados foram coletados por meio de entrevistas individuais no mês de dezembro de 2019. Seguiu-se um roteiro semiestruturado com questões norteadoras e não houve necessidade de repetição. A amostra desta pesquisa foi intencional, sendo a coleta de dados finalizada com 13 entrevistas, diante da saturação dos dados, definida no momento em que novos dados não proporcionavam maiores esclarecimentos para o objeto estudado.

Os dados foram registrados por áudio-gravação e transcritos na íntegra. Foram organizados à luz do Discurso do Sujeito Coletivo (DSC), que orienta a elucidação do pensamento coletivo de determinado grupo social a partir dos posicionamentos dos indivíduos sobre o assunto em questão. Para construção dos DCS, levou-se em consideração as seguintes figuras metodológicas: Expressões-Chave $(\mathrm{ECH})$, Ideia Central (IC) e Ancoragem (AC) ${ }^{(10)}$.

Para discussão dos dados utilizou-se por referencial teórico a Teoria das Representações Sociais (TRS). As representações sociais são fenômenos que se relacionam com a forma singular de compreender e de se comunicar, o qual concebe tanto a realidade quanto o senso comum ${ }^{(11)}$.

Esta pesquisa teve início após aprovação do Comitê de Ética em Pesquisa, sob o protocolo $\mathrm{n}^{\circ}$ 25078719.5.0000.5413, de acordo com a resolução $\mathrm{N}^{\circ} 466 / 2012$ e No510/2016. Todos os participantes receberam as informações pertinentes à mesma por meio do Termo de Consentimento Livre e Esclarecido e, em conformidade com os preceitos éticos, foram representados na pesquisa pela letra P de PARTICIPANTES e, seguido do número ordinal da realização das entrevistas: P1, P2, P3. 
RESULTADOS

Foram entrevistados um total de 13 profissionais, de nível médio a superior. A amostra foi composta por 10 participantes do sexo feminino $(76,92 \%)$ e 3 do sexo masculino $(23,07 \%), 3$ eram enfermeiros $(23,07 \%)$ e 10 eram técnicos de enfermagem (76,92\%). Desse conjunto 7 eram casados (53,84\%), 4 eram solteiros $(30,76 \%)$ e 2 eram divorciados (15,38\%). A média da idade dos participantes foi de 37 anos e o tempo médio na instituição de 7 anos.

A análise dos dados permitiu a elaboração de seis IC, construídas a partir das $\mathrm{ECH}$ das falas dos participantes. Cada IC se apresenta acompanhada do seu discurso, que foram organizados conforme as questões norteadoras, como demonstra o Quadro 1.

\section{DISCUSSÃO}

Os dados da pesquisa discorreram sobre os cuidados de uma equipe de enfermagem com práticas empíricas no que diz respeito ao atendimento de paciente politraumatizado. A primeira ideia central retrata sobre a priorização da estabilidade hemodinâmica do paciente.
O protocolo ATLS institui que a primeira ação a ser feita é a preparação e organização do local de atendimento além dos materiais, equipe de funcionários e Equipamentos de Proteção Individual ${ }^{(12)}$. No que tange a sequência do atendimento, avalia-se o paciente levantando suas prioridades de tratamento no seguimento: manutenção das vias aéreas e restrição da movimentação da coluna cervical, respiração e ventilação, circulação com controle de hemorragia, avaliação do estado neurológico e exposição ambiental (13).

O primeiro discurso construído a partir das falas dos participantes repor-

\section{QUADRO 1- Ideias centrais e respectivos discursos de cada questão norteadora. Marília/SP, Brasil, 2019.}

Quando chega um paciente politraumatizado, como se dá sua prática nesse atendimento?

IC 1- No atendimento do paciente politraumatizado, a equipe de enfermagem prioriza sua estabilidade hemodinâmica

Fale sobre o(s) protocolo(s) para atendimento de paciente politraumatizado utilizado(s) na Instituição que você trabalha.

IC 2- Desconhecimento da equipe de enfermagem sobre protocolo para atendimento do paciente politraumatizado
DSC 1-A gente vê os sinais vitais, nível de consciência, faz a monitorização, prioriza a cervical e a prancha rígida, vê como vamos tirar a prancha, auxilia o médico para virar para palpar a coluna, com movimentação em bloco, vê se vai tirar ou manter o colar. Além disso, puncionar uma veia calibrosa, aguarda a conduta do médico, vai seguindo o que o doutor vai pedindo, se ele tá com muita dor a gente vai medicar, faz analgesia ou se tiver alguma coisa pra estancar a gente estanca e encaminha para o setor de imagem, pede o raio- $\mathrm{X}$, faz tomografia e colhe exames laboratoriais (P1, P2, P3, P4, P5, P6, P8, P9, P10, P11)

DSC 2- Eu não sei dizer se tem ou não, nunca fomos orientados para isso, na sala acho que não tem nenhum, pelo nosso conhecimento, não que eu saiba, se tem eu não sei, Nunca teve uma educação continuada em relação a isso aí, se tiver, nos é desconhecido. (P2, P3, P4, P7, P9 e P10)

DSC 3- Na instituição tem protocolo de trauma, acredito que é próprio da instituição, a gente tem os protocolos que ficam arquivados, tem pra medicina a gente segue esse protocolo, entre aspas, que é o que a gente faz em todo paciente (P1, P5, P6 e P8)

Você participou da elaboração de protocolos ou participou de algum treinamento para atender um paciente politraumatizado na sua instituição? () Sim () Não. Se sim, fale como foi isso pra você.

IC 3- Participação da equipe de enfermagem na elaboração de protocolos para atendimento de pacientes politraumatizados

IC 4- Participação da equipe de enfermagem em capacitações diversificadas

Qual sua opinião sobre a elaboração de protocolos e treinamentos institucionais sobre 0 atendimento ao paciente politraumatizado?

DSC 4- Não, já teve treinamento para parada cardíaca, fiquei um mês em treinamento né, a gente fica com uma pessoa que já sabe, capacitações eles são mais, né, a gente dez alguns treinamento, pra trabalhar na instituição, mas para politraumatizado, não. (P2, P7, P8, P9 e P10)
IC 5- Os protocolos qualificam a assistência de enfermagem

IC 6- Os protocolos não beneficiam a ação da enfermagem
DSC 5-Acho importante porque esquematiza o processo de trabalho, é para o nosso conhecimento, para gente trabalhar da maneira certa. Por isso, deveria ter mais treinamento e capacitações, eu acho importante treinar a equipe para atender bem o paciente, ter mais destreza, mais firmeza na hora de fazer as coisas poderia ser mais rápido se todo mundo falar a mesma língua, você já vê e já sabe o que tem que fazer, não precisa ficar esperando o médico ( $\mathrm{P} 1$, P2, P3, P4, P5, P7, P8 e P9)

DSC 6- Eu acho que apra enfermagem não ajudaria, não tem tempo de ficar estudando protocolos todo dia (P10 e P11) 
ta ações fragmentadas, destoante do que se preconiza no protocolo ATLS e, a equipe de enfermagem se coloca em cumprimento de determinações médicas. Por isso, vemos ações baseadas no que elas visualizam de outros colegas, sem arcabouço teórico.

Segundo os pressupostos da TRS, o indivíduo reage com ações que estejam fortemente ligadas ao local onde convivem cotidianamente, essas representações, que são partilhadas por tantos, penetram e influencia a mente de cada um, elas não são pensadas por eles, são repensadas, recitadas e reapresentadas ${ }^{(11)}$.

Os protocolos, além de fornecerem base científica, direcionam as ações que devem ser realizadas para o bom atendimento do paciente, sendo executadas em caráter complementar e interdisciplinar. Estudo desenvolvido com a equipe de enfermagem em um serviço de emergência de Santa Catarina evidenciou que a equipe não dispõe de um protocolo para o atendimento ao paciente vítima de trauma e ainda identifica que na avaliação primária, a maioria cita o ABCDE como regra para atendimento, mas não sabem definir as ações propostas nas etapas. Tais evidencias congraçam com os achados neste estudo, levando em consideração a segunda ideia central que relatou sobre o desconhecimento da equipe de enfermagem sobre os protocolos institucionais utilizados para o atendimento de paciente politraumatizado $^{(14)}$.

O segundo e terceiro discurso ecoam sobre a inexistência de protocolos para politraumatismo na sala de emergência. Os participantes afirmam que se há protocolos instituídos, estes não foram divulgados, o que reforça a ideia de ações empíricas. Depreende-se assim que, apesar de realizarem um seguimento de condutas e procedimentos, a equipe de enfermagem não apresenta clareza da significação do termo protocolo nem de suas ações, o que vai ao encontro da TRS, onde os fenômenos específicos relacionados a um modo particular de compreender e se comunicar, cria tanto a realidade, quanto o senso comum ${ }^{(11)}$.

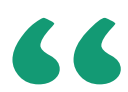

\section{Protocolo de atendimento} engloba informações que auxiliam no direcionamento do trabalho, estimulando a visão crítica dos profissionais colaborando no desempenho de capacidade de comportamento individual e coletivo.

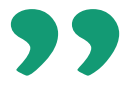

O protocolo de atendimento engloba informações que auxiliam no direcionamento do trabalho, estimulando a visão crítica dos profissionais colaborando no desempenho de capacidade de comportamento individual e coletivo. Sendo que, quando a equipe não pratica o segmento de ações preconizadas através de um protocolo a eficácia da assistência diminui (14).

A implementação de protocolos demanda muito além do cuidado assistencial, para isso, deve-se considerar também que os profissionais adquiram um embasamento teórico mediante capacitações teórico-práticas continuadas, o conhecimento científico fundamenta o raciocínio clínico e apresenta o intuito de presumir a dinâmica dos sistemas corporais dos pacientes politraumatizados e possíveis complicações ${ }^{(15)}$.

Diante da necessidade de incorporar práticas pautadas em ciência, direcionada por protocolos, idealiza-se que a equipe de enfermagem esteja engajada nesse processo, desde sua construção, até mesmo à aplicação prática. No entanto, a terceira e quarta ideia central reafirmam que a equipe de enfermagem da instituição em cena, não participou da elaboração de protocolos e ainda, relata sobre capacitações diversas, exceto ao atendimento de politraumatizados, conforme descrito no quarto discurso.

A equipe de enfermagem representa o maior percentual de profissionais na equipe de saúde e sua prática consequentemente reflete na qualificação do serviço. É preciso que se tenha Educação Permanente associada à Prática Baseada em Evidências (PBE) e linguagem padronizada para garantir uma melhor assistência aos pacientes, além de acrescentar valorização da profissão ao demonstrar o domínio das bases científicas do cuidado, e, proporcionar um desenvolvimento profissional ${ }^{(16)}$.

Nessa lógica, a quinta ideia central trouxe a consciência da equipe de enfermagem em reconhecer que, os protocolos, capacitações e treinamentos favorecem tanto os profissionais quanto o paciente, ressaltando o aprimoramento do conhecimento técnico-científico e a oferta de um cuidado mais adequado. No quinto discurso construído, os profissionais exprimiram um sentimento de fragilidade por parte da disponibilização institucional, e mostraram-se carecidos em participar de treinamentos ou meios que 
dispusessem atualizações do conhecimento e aperfeiçoamento na destreza técnica, indicando uma gestão vertical do processo de trabalho.

Um estudo realizado na Austrália evidenciou que a equipe de enfermagem, após a aplicação de um módulo de avaliação de pacientes, apresentou evolução no conhecimento e auto eficácia no atendimento, indicando que a educação permanente e oportunidades de recursos apropriados são essenciais para melhorar a performance desses profissionais. Nessa vertente, as colocações do quinto discurso afiançaram com a literatura, pois os protocolos e capacitações ajudam no processo de trabalho, oferecem apoio na padronização do atendimento e consequentemente no cuidado direto e indireto ao paciente ${ }^{(4)}$.

Divergências foram transcorridas na sexta ideia central, onde dois participantes ressaltaram que o protocolo não é um fator positivo para equipe de enfermagem, destacando que o tempo seria um empecilho para estudá-lo. No entanto, um estudo realizado em 51 unidades de saúde e no SAMU de uma cidade no interior paulista, apontou potencialidades na aplicação de protocolos como a segurança do paciente, a segurança no desenvolvimento das intervenções, a atualização de procedimentos e o fácil entendimento, indicando numerosas vantagens em sua implementação, contrapondo o sexto discurso apresentado na pesquisa ${ }^{(16)}$.

As teorias de Enfermagem foram desenvolvidas com o intuito de aprimorar a prática por intermédio de seu uso pelos profissionais, visando retratar, elucidar, predizer ou prescrever o fenômeno do cuidado de Enfermagem ${ }^{(17)}$. O fato de os discursos exprimirem a atuação desses profissionais com embasamento empírico, seguindo a conduta de outros colegas, mostra um retrocesso na conquista da enfermagem como ciência. Sem o benefício dos instrumentos científicos, tendemos a considerar e analisar o mundo de uma maneira semelhante; especialmente quando o mundo em que vivemos é totalmente social, suscitando na banalização da prática do trabalho baseada em senso comum ${ }^{(11)}$.

\section{CONCLUSÃO}

Levando em consideração o objetivo da pesquisa, foi possível identificar que os profissionais de enfermagem conseguem contemplar diversas etapas do ABCDE do trauma preconizados pelo ATLS utilizado na instituição, entretanto, não o perfilham como um protocolo, e sim como prática habitual do cotidiano adquirida através da transferência de informações por outros profissionais. Todavia, reconhecem a importância de recursos como capacitações e treinamentos para aprimorar o conhecimento técnico-científico.

A presente pesquisa foi desenvolvida em um único serviço e urgência e emergência do interior paulista e contou com apenas 13 participantes, o que se caracteriza como limitações para estudo. No entanto, os achados destacam a importância de cuidados de enfermagem devidamente direcionados por bases científicas, favorecendo uma prática profícua e segura ao paciente politraumatizado.

\section{Referências}

1. Lentsck MH, Oliveira RR, Corona LP, Mathias TA. Fatores de risco para óbito de pacientes com trauma internados em Unidade de Terapia Intensiva. Rev Latino-Am Enfermagem. 2020;28(e3236):1-12.

2. Gomes ATL, Ferreira Jr MA, Salvador PTCO, Bezerril MS, Santos VE. Segurança do paciente em situação de emergência: percepções da equipe de enfermagem. Rev Bras Enferm. 2019;72(3):753-9.

3. Padilha KG, Barbosa LR, Andolhe R, Oliveira EM, Ducci AJ, Bregalda RS, Secco LMD. Carga de trabalho de enfermagem, estresse/burnout, satisfação e incidentes em unidade de terapia intensiva de trauma. Texto Contexto Enferm. 2017;26(3):e1720016.

4. Curtis K, Wiseman T, Kennedy B, Okorocha S, Goldsmith H. Implementation and evaluation of ward-based learning program of trauma patient management. $J$ Trauma Nurse. 2016;23(1):28-35.

5. Battist GR, Branco A, Caregnato RCA, Oliveira MMC. Perfil de atendimento e satisfação dos usuários do Serviço de Atendimento Móvel de Urgência (SAMU). Rev Gaúcha Enferm. 2019;40:e20180431.

6. Schweitzer G, Nascimento ER, Malfussi LBH, Hermida PMV, Nascimento KC, Moreira AR. Implementation of the protocol of nursing care in trauma in aeromedical service. Rev Bras Enferm. 2020;73(3):e20180516.

7. Wahlin RR, Lindstrom V, Ponzer S, Vicente V. Patients with head trauma: a study on initial prehospital assessment and care. Int Emerg Nurs. 2018;36:51-5.

8. Tong A, Sainsbury P, Craig J. Critérios consolidados para relatar pesquisas qualitativas (COREQ): uma lista de verificação de 32 itens para entrevistas e grupos de foco. Int J Qual Health Care. 2007; 19(6):349-57.

9. Faculdade de Medicina de Marília. Nossa história. Marília (SP): Famema; c2018 [citado 3 out 2019]. Disponível em: http://hc.famema.br/nossa-historia.

10. LEFREVE F, LEFREVE AMC. Discurso do sujeito coletivo: representações sociais e intervenções comunicativas. Texto \& Contexto Enferm. 2014;23(2):502-7. 11. Moscovici S. Representações sociais: investigações em psicologia social. $11^{\text {a }}$ ed. Petrópolis (RJ): Vozes; 2015.404 p.

12. The Committee on Trauma. ATLS Advanced Trauma Life Support: student course manual. 10th ed. Chicago: American College of Surgeons; 2018.

13. Perboni JS, Silva RC, Oliveira SG. A humanização do cuidado na emergência na perspectiva de enfermeiros: enfoque no paciente politraumatizado. Interações. 2019;20(3):959-72

14. Mattos $L S$, Silvério MR. Avaliação do indivíduo vítima de politramatismo pela equipe de enfermagem em um serviço de emergência de Santa Catarina. Rev Bras Promoç Saúde. 2012;25(2):182-91.

15. Madureira VS. Os saberes da enfermagem. Rev Bras Enferm. 2004;57(3):357-60.

16. Sales CB, Bernardes A, Gabriel CS, Brito MFP, Moura AA, Zanetti ACB. Standard operational protocols in professional nursing practice: use, weaknesses and potentialities. Rev Bras Enferm. 2018;71(1):126-34.

17. Dias JA, David HM, Vargens OM. Ciência, enfermagem e pensamento crítico: reflexões epistemológicas. Rev Enferm UFPE. 2016;10(4):3669-75. 